\title{
Deterministic Laws and Epistemic Chances
}

\author{
Wayne C. Myrvold \\ Department of Philosophy \\ The University of Western Ontario \\ wmyrvold@uwo.ca
}

\begin{abstract}
In this paper, a concept of chance is introduced that is compatible with deterministic physical laws, yet does justice to our use of chance-talk in connection with typical games of chance, and in classical statistical mechanics. We take our cue from what Poincaré called "the method of arbitrary functions," and elaborate upon a suggestion made by Savage in connection with this. Comparison is made between this notion of chance, and David Lewis' conception.
\end{abstract}

\section{Probability, Chance, and Credence: a brief history}

As has been often pointed out, the word "probability" has been used in at least two distinct senses. ${ }^{1}$ One sense, the epistemic sense, has to do with degrees of belief of a rational agent. The other sense, which Hacking calls the aleatory sense, is the concept appropriate to games of chance; this is the sense in which one speaks, for example, of the probability (whether known by anyone or not) of rolling at least one pair of sixes, in 24 throws of a pair of fair dice.

A particularly clear statement that there are two concepts that need to be distinguished is found in Poisson's book of 1837.

In ordinary language, the words chance and probability are nearly synonymous. Quite often we employ one or the other indifferently, but when it is necessary to distinguish between their senses, we will, in this work, relate the word chance to events in themselves, independently of our knowledge of them, and we will reserve for the word probability the previous [epistemic] definition. Thus, an event will have, by its nature, a greater or less chance, known or unknown; and its probability will be relative to the knowledge we have, in regard to it.

\footnotetext{
${ }^{1}$ See Hacking (1975) for a masterful overview of the history.
} 
For example, in the game of heads and tails, ${ }^{2}$ the chance of getting heads, and that of getting tails, results from the constitution of the coin that one tosses; one can regard it as physically impossible that the chance of one be equal to that of the other; nevertheless, if the constitution of the coin being tossed is unknown to us, and if we have not already subjected it to trials, the probability of getting heads is, for us, absolutely the same as that of getting tails; we have, in effect, no reason to believe more in one than the other of the two events (Poisson, 1837, p. 31). ${ }^{3}$

Note that Poisson's use of "chance" refers to single events, and the chance of heads on a coin toss is a matter of the physical constitution of the chance set-up (he says "the constitution of the coin," but clearly it matters also how the coin is tossed). This is not a frequency interpretation.

Something happened on the way to the twentieth century: the notion of objective chance - that is, single-case probability thought of as a feature of a physical situationlargely dropped out of discussions. There remained that the idea that "probability" can be taken in either an objective or an epistemic sense, but the objective sense became identified with a frequency interpretation.

Thus we find de Finetti rejecting objective notions of probability on the basis of a rejection of Laplacean and frequency conceptions (de Finetti 1937, pp. 16ff; 1980, pp. 71ff). In a similar vein, Savage $(1972$, p. 4) identifies an objective conception of probability with a frequency interpretation, and takes it as a virtue of the subjectivist view that it is applicable to single cases. Absence of the notion of objective chance in so many discussions of the foundations of probability led Popper $(1957,1959)$ to conclude that he had an entirely new idea in single-case objective probabilities, which he called "propensities."

We also find some historians of probability have projecting a frequency conception onto writers of previous centuries, in place of the notion of chance. For example, though Hacking $(1975$, p. 12) initially characterizes this duality much as we have

\footnotetext{
${ }^{2}$ Poisson says "croix et pile"; heads and tails is our equivalent.

${ }^{3}$ Dans la langage ordinaire, les mots chance et probabilité sont à peu près synonymes. Le plus souvent nous emploierons indifféremment l'un et l'autre; mais lorsqu'il sera nécessaire de mettre une différence entre leurs acceptions, on rapportera, dans cet ouvrage, le mot chances aux événements en eux-mêmes et indépendamment de la connaissance que nous en avons, et l'on conservera au mot probabilité sa définition précédente. Ainsi, un événement aura, par sa nature, une chance plus ou moins grande, connue ou inconnue; et sa probabilité sera relative à nos connaissances, en ce qui le concerne.

Par exemple, au jeu de croix et pile, la chance de l'arrivée de croix et celle de l'arrivée de pile, résultent de la constitution de la pièce que l'on projette ; on peut regarder comme physiquement impossible que l'une de ces chances soit égale l'autre; cependant, si la constitution du projectile nous est inconnue, et si nous ne l'avons pas déjà soumis à des épreuves, la probabilité de l'arrivée de croix est, pour nous, absolument la même que celle de l'arrivée de pile: nous n'avons, en effet, aucune raison de croire plutôt à l'un qu'à l'autre de ces deux événements. I'l n'en est plus de même, quand la pièce a été projetée plusieurs fois: la chance propre à chaque face ne change pas pendant les épreuves; mais, pour quelqu'un qui en connaît le résultat, la probabilit de l'arrivée future de croix ou de pile, varie avec les nombres de fois ces deux faces se sont déjà présentées.
} 
here, later in the book the aleatory concept of probability identified with a frequency interpretation: "On the one hand it [probability] is epistemological, having to do with support by evidence. On the other hand it is statistical, having to do with stable frequencies" (p. 43). Hacking is far from alone in this. For example, Daston (1988, p. 191) attributes a frequency notion to A. A. Cournot (1843), who distinguishes between probability and what he calls degrees of physical possibility in much the same way that Poisson distinguished between probability and chance. Howie (2002, p. 36) attributes a frequency concept of chance to Poisson.

It's not entirely clear what the reason is for the disappearance of chance from discussions of probability. A plausible conjecture is that it had to do with increasing acceptance that the laws that govern the physical world are deterministic, together with the notion that objective chance and determinism are incompatible. Laplace famously began his Philosophical Essay on Probabilities (1814) with a discussion of determinism. A being that had complete knowledge of the laws of nature and the state of the world at some time, and was able to perform the requisite calculations, would have no need of the calculus of probabilities, according to Laplace. It is only because our abilities depart from those of such a being that we employ probabilities. Laplace then proceeds to characterize probability in epistemic terms; "[ $t$ ]he theory of chance consists in reducing all the events of the same kind to a certain number of cases equally possible," where "equally possible" means that we are equally undecided about which of the cases obtain (Laplace, 1951, p. 6). It is not clear that Laplace is consistent in maintaining an epistemic view throughout; there are passages in the Essay that suggest that it is a matter of fact, which we can investigate empirically, whether events are truly equipossible. Nevertheless, the official view, for Laplace, is an epistemic one. ${ }^{4}$

Though it may be that emphasis on determinism led to the decay of the notion of objective chance, there is nevertheless a tension between the idea that chance and determinism are incompatible, and the way that we talk about chances. We talk about the chance of heads on a coin toss, casino owners worry about whether their roulette wheels show discernible bias, and these seem to be matters that can subjected to experimental test, by doing multiple trials and performing a statistical analysis on the results. ${ }^{5}$ Yet we also think that, at least to the level of approximation required, these systems can be adequately modelled by deterministic, classical physics. ${ }^{6}$ Nor does it seem that Poisson was supposing any departure from determinism.

Of course, such talk might simply be deeply ill-conceived. David Lewis declared that "[t]o the question how chance can be reconciled with determinism, ... my answer is: it can't be done" (1986, p. 118), and Schaffer (2007) has provided arguments for this conclusion. It is the purpose of this paper to present a notion that may appropriately be called chance, which fits quite nicely with deterministic evolution of a certain kind, and which is suited to play the role of chance in our chance-talk. Schaffer

\footnotetext{
${ }^{4}$ See Hacking $(1971 ; 1975$, Ch. 14) for a lucid discussion.

${ }^{5}$ Though perhaps this should go without saying, it should be emphasized that taking frequency data as evidence about chances is not tantamount to holding a frequency interpretation of chance.

${ }^{6}$ Though, it must be noted, Lewis (1986, p. 119) has suggested that this is false.
} 
(2007) distinguishes between genuinely objective chances and what he calls "epistemic chances," while noting that the latter can be objectively informed. "Epistemic chance" is an apt name for the notion that we will be introducing, as it highlights the fact that we will be interweaving epistemic and physical considerations.

In what follows, we will use the word "chance" for the aleatory concept; a chance is an objective, single-case probability. When we are speaking of the belief states of a (possibly idealized) agent, we will us the word "credence." "Probability" will be used when we want to be noncommittal.

\section{Learning about chances}

The chance of heads on a coin toss, if it is regarded as an objective feature of the set-up, is ipso facto the sort of thing that we can have beliefs about, beliefs that may be correct or incorrect, better or worse informed. Under certain conditions, we can learn about the values of chances.

Particularly conducive to learning about chances are cases in which we have available (or can create) a series of events that we take to be similar in all aspects relevant to their chances, that are, moreover, independent of each other, in the sense that occurrence of one does not affect the chance of the others. The paradigm cases are the occurrence of heads on multiple tosses of the same coin, occurrence of a six on multiple throws of the same die, and the like. Consider a sequence of $N$ coin tosses. If, on each toss, the chance of heads is $\lambda$, then the chance of any given sequence of results is

$$
\lambda^{m}(1-\lambda)^{N-m},
$$

where $m$ is the number of heads in the sequence. Considered as a function of $\lambda$, this is peaked at the observed relative frequency $m / N$, and becomes more sharply peaked, as $N$ is increased.

Let $E$ be the proposition that expresses the sequence of results these $N$ tosses, and, for any $\lambda$, let $H_{\lambda}$ be the proposition that the chance of heads on each toss is equal to $\lambda$. Consider an agent who has some prior credences about the chance of heads, and updates them by Bayesian conditionalization:

$$
\operatorname{cr}\left(H_{\lambda}\right) \Rightarrow \operatorname{cr}\left(H_{\lambda} \mid E\right)=\frac{\operatorname{cr}\left(E \mid H_{\lambda}\right) \operatorname{cr}\left(H_{\lambda}\right)}{\operatorname{cr}(E)} .
$$

It seems natural to suppose - and, indeed, in the statistical literature this is typically assumed without explicit mention - that our agent's credences set $\operatorname{cr}\left(E \mid H_{\lambda}\right)$ equal to the chance of $E$ according to $H_{\lambda}$, as is required by what Lewis (1980) has dubbed the Principal Principle. This has the consequence that our agent's credence in chancevalues close to the observed relative frequency is boosted, and her credence in other values, diminished. Moreover, since the likelihood function $\lambda^{m}(1-\lambda)^{N-m}$ is more sharply peaked, the larger the number of trials, relative frequency data becomes more valuable for narrowing credence about chances as the number of trials is increased. 
Note that there are three distinct concepts at play here: chance, credence, and relative frequency in repeated trials. None of these three is to be identified with any of the others. They do, however, connect in a significant way: relative frequency data furnish evidence on which we update credences about chances.

\section{3 "Almost Objective" Chances}

We take our cue from what Poincaré called the method of arbitrary functions (see von Plato (1983) for the history of this). Poincaré's analysis leaves some crucial questions unanswered, and, in particular, leaves it unclear what notion of probability might be in play. Savage (1973) argued that subjective credence has a role to play. And so it does, but it is not a radical subjectivism that is needed, but a tempered personalism that distinguishes between reasonable and unreasonable credence-functions.

\subsection{Tempered personalism about credences}

Objective Bayesians hold that there are, for any body of background knowledge, unique credences that would be the degrees of belief of an ideally rational agent. At the opposite extreme would be radical subjectivists (if there are any), who hold that the only constraint on credences is the requirement of coherence, that is, that they satisfy the axioms of probability; within the class of coherent credence functions, there can be no grounds for judging one better or worse than another. ${ }^{7}$

The attitude that Abner Shimony (1971) has dubbed tempered personalism steers between these extremes, finding cognitive virtue between two opposing vices. Without supposing that there are uniquely rational credences, not all are equally acceptable; excessively dogmatic credences are to be eschewed as obstacles to learning about the world. In what follows, we will assume that there is a class of credences, perhaps imprecisely defined, that represent the possible credences of a reasonable agent.

As an example relevant to the sorts of cases we are discussing, suppose that a gambler in a casino becomes convinced that the next spin of the roulette wheel will be 23 , to the extent that he is willing to bet his life savings on it. ${ }^{8}$ Not because he thinks the game is rigged; he takes it to be an ordinary roulette wheel, being spun in the ordinary way, with the ordinary sorts of causal influences on the outcome. Though the gambler's credences need not violate coherence, we would nevertheless take such a conviction to be unreasonable. If, further, our gambler claimed that his conviction was based on a belief that the current state of everything causally relevant to the outcome was such as to lead, via the unfolding of deterministic laws of physics, to the result 23, we would, even if we shared his conviction in determinism, nevertheless regard it as ludicrous to pretend to knowledge to the degree of precision that such a

\footnotetext{
${ }^{7}$ The parenthetical qualification is due to the fact that, though, in some passages de Finetti sounds like a radical subjectivist, there are others that indicate a more moderate position.

${ }^{8}$ This example is inspired by the behaviour of the character played by James Garner in the comic western Support Your Local Gunfighter (1971).
} 
conviction would require. In what follows, I will simply take it that the reader shares this judgment, with the issue of what justification we might have for this to be left for another occasion.

\subsection{Poincaré and the method of arbitrary functions}

Poincaré $(1912,1952)$ considered a simple, roulette-like game, in which a wheel, divided into a large number $n$ of sectors of equal size, alternately colored red and black, is spun, and eventually comes to rest due to friction. Bets are to be placed on whether a pointer affixed to the wheel's mount will point to a red or a black sector when the wheel comes to rest. The set-up is such that small differences in the initial impulse, too small to be perceived or controlled, can make a difference between the outcome being red or black.

Poincaré supposes the probabilities of initial impulses to be given by a density function $\phi$, a function that is "entirely unknown" (1912, p. 148). This function yields, via the dynamics of the set-up, a density function $f$ over the angle $\theta$ at which the wheel comes to rest. Suppose, now, that the function $f$ is continuous and differentiable, and that the derivative is bounded, so that, for some $M,\left|f^{\prime}(\theta)\right|<M$ for all $\theta$. If the angle (in radians) subtended by each sector is $\epsilon$, then the difference between the probability of red and the probability of black is at most $M \pi \epsilon$. This goes to zero as $\epsilon$ goes to zero.

There are a number of questions left open by Poincaré's discussion. First is the status of the limit $\epsilon \rightarrow 0$. We are, after all, considering the probability of a red or black outcome on a spin of a particular wheel, with fixed number of sectors, not a sequence of wheels with an ever-increasing number of sectors. We need not take Poincaré's limittalk literally. What matters is that $f$ vary slowly enough over intervals of size $\epsilon$ that the probability of landing in any red sector be approximately equal to the probability of landing in the adjacent black sector, and that any differences between probabilities associated with successive sectors be small enough that they remain negligible when $n / 2$ of them are summed.

For our purposes, a more serious issue is the status of the function $\phi$, which yields probabilities over initial conditions. Poincaré calls $\phi$ an unknown function, which suggests that there is a matter of fact about what function it actually is. In a discussion of the game of roulette, Poincaré writes,

What is the probability that the impulse has this or that value? About this I know nothing, but it is difficult for me not to admit that the probability is represented by a continuous analytic function (Poincaré, 1912, p. 12). ${ }^{9}$

In a parallel discussion in Science and Hypothesis, he writes, ${ }^{10}$

I do not know what is the probability that the ball is spun with such a force that this angle should lie between $\theta$ and $\theta+d \theta$, but I can make a convention.

\footnotetext{
${ }^{9}$ Quelle est la probabilité pour que cette impulsion ait telle ou telle valeur? Je n'en sais rien, mais il m'est difficile de ne pas admettre que cette probabilité est representée par une fonction analytique continue.

${ }^{10}$ Note that there is a shift of notation between Calcul des Probabilités and Science and Hypothesis; $\phi$ is here a density function over the final angle, that is, the function we have been calling $f$.
} 
I can suppose that this probability is $\phi(\theta)$. As for the function $\phi(\theta)$, I can choose it in an entirely arbitrary manner. I have nothing to guide me in my choice, but I am naturally induced to suppose the function to be continuous (Poincaré, 1952, p. 201).

This, it must be admitted, is puzzling. Poincaré alternates between treating the probability as something objective but unknown, and treating it as something that we can make arbitrary choices about.

Suppose, now, that we take the function $\phi$ to represent an agent's degrees of belief about the impulse imparted to the wheel. Applying the dynamics of the system to this credence function yields a probability density $f$ over the orientation of the wheel after it has come to rest, at some later time $t_{1}$. The probability function yielded by $f$ might not represent our agent's degrees of belief about the final angle, if she doesn't know the dynamics of the set-up, or is unable to perform the requisite calculation; this will be important in $\S 3.3$, below.

Suppose, now, that small changes in the initial conditions - too small to be controlled or noticed by our agent-yield differences in the final angle that are large compared to the width of a single sector. It is reasonable to suppose that an agent's credences would not vary much over such small scales; a credence function that changed appreciably when shifted by an imperceptible amount would represent more detailed knowledge of initial conditions than would be available to an agent in the epistemic situation we are imagining. Then application of the system's dynamics to the agent's credences will yield roughly equally probabilities of red and black outcomes. Moreover, this conclusion does not depend sensitively on the function $\phi$. Though, pace Poincaré, it is not true that an arbitrary probability density over initial conditions, or even an arbitrary density with bounded variation, yields equal probabilities for red and black, it is true that a mild constraint on the density function $\phi$-moreover, a constraint that arguably any reasonable credence should satisfy - suffices to entail that $f$ yield approximately equal probabilities for red and black. The dynamics of the set-up ensure that any reasonable credences about states of affairs at one time yield approximately the same probabilities for certain coarse-grained propositions about a later state of the system.

Generalizing, the situations of interest to us are ones in which we have a physical set-up such that, for some proposition $A$ (or class of propositions) about the outcome of an observation undertaken at time $t_{1}$, the dynamics of the set-up are such that any reasonable credences about states of affairs at time $t_{0}$ yield, as a result of the evolution of the system, approximately the same value for the probability of $A$. There are four interacting components at play here. One is a limitation on the knowledge of the system available to the agent. Although it remains true that a being in possession of precise knowledge of initial conditions and able to do the requisite calculation would be in a position to have precise knowledge about the outcome, we suppose limits to the precision of the knowledge available to our agents. Second is a judgment about what sorts of credences are reasonable, given the knowledge available to our agents; we are neither supposing uniquely reasonable credences, nor are we supposing that 
coherence is the only criterion of reasonableness. Third is a limitation of attention to certain macroscopic propositions about the system's state at a later time. Lastly, and crucially, it should be a feature of the dynamics of the system that any differences between reasonable credence-functions wash out; any reasonable credences about initial conditions lead to approximately the same credence in the proposition $A$.

In situations like this - in which the dynamics of the system lead all reasonable credences about the state of affairs at $t_{0}$ to effectively the same probability for some proposition $A$ about states of affairs at a later time $t_{1}$-we are justified, I think, in calling this common probability the chance of $A$. What value these chances have depends on the physics of the set-up, and, moreover, it makes sense to talk of unknown chances, or in cases of disagreement about what the chances are, about one value being more correct than another. The limitations on knowledge might be in principle limitations. For an example that may or may not apply to the real world, consider the de Broglie-Bohm hidden-variable interpretation of quantum mechanics. There, it is provably impossible for an agent (who must interact with a system via physical means to gain information about it) to gain enough information about corpuscle positions to make betting at other than the quantum-mechanical probabilities reasonable. In other cases, even if it might in principle be possible to gain further knowledge, obtaining such knowledge is so far beyond feasibility that it might as well be impossible in principle. Consider a real roulette wheel, to be spun by a human croupier, and take $t_{0}$ to be some time before the spin. ${ }^{11}$ Even if it is possible, in principle, to gain sufficient information about the croupier's physical state and all influences on it that would be sufficient to make it reasonable to bet at other than the values we are calling chances, this matters little to the credences of actual agents. Quantities of this sort have been called "almost objective" (Machina, 2004). On the issue of terminology, see $\S 5$, below.

\subsection{Learning about chances, revisited}

The function $\phi$ is meant to represent an agent's credences about states of affairs at $t_{0}$; $f$, the result of applying the system's dynamics to this function. These dynamics will often be imperfectly known to the agent, who might be unsure, say, whether the wheel is biased in some way. Even if the dynamics are known, the requisite computation might be intractable.

Nonetheless, our agent might believe that there is some value, unknown to her, that gives the probability assigned to a proposition $A$ by time-evolving, not only her current credences, but those of any reasonable agent. This value is the degree of belief in $A$ that a reasonable agent would have if she knew the dynamics of the set-up and could do the calculation, and in this sense represents credence that makes optimal use of information available. Our agent can have credences about what this value is. For any real number $\lambda$, let $H_{\lambda}$ be the proposition that this value is equal to $\lambda$. It is a reasonable constraint on our agent's credences that they satisfy

$$
\operatorname{cr}\left(A \mid H_{\lambda}\right)=\lambda,
$$

${ }^{11}$ After the ball has been released is another matter. 
and that, moreover, if $E$ is any proposition whose truth-value could be ascertained by the agent at $t_{0}$,

$$
\operatorname{cr}\left(A \mid H_{\lambda} \& E\right)=\lambda .
$$

To see that this is a reasonable condition on an agent's credences, recall that, if $\lambda$ is the chance of $A$, and our agent's credences in $A$ is not equal to $\lambda$, this is due to the agent's imperfect knowledge of the dynamical laws governing the system, or else to her inability to apply these laws. Her conditional credence, conditional on the supposition that her credence would be $\lambda$ were these limitations lifted, is required to be $\lambda$.

Our constraint on credence suffices for our agent to learn about the chances of a series of events that are regarded as having equal and independent chances, in the manner outlined in section 2, above. The constraint is a cousin of Lewis' Principal Principle. The chief difference is that, in the Principal Principle, $E$ may be any $a d-$ missible proposition, and, though Lewis does not explicitly define admissibility, he takes all statements about the past to be admissible. This would be unjustified on our treatment.

There is considerable literature on the justification of the Principal Principle; to some it appears a mysterious constraint. ${ }^{12}$ About our constraint there is no mystery.

\section{Chances in statistical mechanics}

An appropriate physical set-up can wash out very considerable differences in credences about initial conditions. Consider a gas in a box with with a partition down the middle. Alice believes that at $t_{0}$ the gas is initially in the left side of the box; Bob, that it is in the right. The partition is removed, and, a few minutes later, at $t_{1}$, some measurements are to be performed on the gas. Let $\phi^{A}\left(t_{0}\right)$ and $\phi^{B}\left(t_{0}\right)$ be Alice and Bob's credences about the state of the system at $t_{0}$, and let $\phi^{A}\left(t_{1}\right)$ and $\phi^{B}\left(t_{1}\right)$ be the result of applying the actual dynamical evolution of the system to these credence-functions. That is, the probability assigned by $\phi^{A}\left(t_{1}\right)$ to a region $\Delta$ of the system's phase space is the probability that $\phi^{A}\left(t_{0}\right)$ assigns to the system being at $t_{0}$ in some state that will evolve into a state in $\Delta .{ }^{13}$ Though this would be difficult to prove rigorously for anything like a realistic gas, there is good reason to believe that, provided that their initial credences don't vary too rapidly within the respective regions of phase space on which they are non-zero, the probability functions that result from applying the dynamics of the system to Alice's and Bob's credences about initial conditions will yield virtually the same probabilities for the results of any feasible measurements (that is, there is no feasible experiment to be performed on the gas that will be informative about whether, a few minutes earlier, the gas had been in the left or the right side of the box). This

\footnotetext{
${ }^{12}$ For some references to this literature, and skepticism about the possibility of a cogent justification, see Strevens (1999).

${ }^{13}$ Once again, these time-evolved credence functions might not be Alice and Bob's credences about the states of affairs at $t_{1}$, if they don't know the dynamics of the system, or are unable to do the requisite calculation.
} 
is true even though, in one sense, the time-evolved credences are as different as the original ones: if there is no overlap between the regions of phase space that Bob and Alice believe the gas to be in at $t_{0}$, there will be no overlap in the regions assigned nonzero probability by the time-evolved credence functions. Nevertheless, these two regions will be finely intertwined in the phase space of the system, and macroscopic regions will contain roughly equal proportions of both, so that the two probability functions will agree closely on the probabilities of outcomes of macroscopic observations.

Here we see a role to play for the equilibrium probability measures used in statistical mechanics. Provided the relaxation to the new equilibrium proceeds as we think it does, Alice's and Bob's time-evolved credences about measurements will not only agree with each other, but with those of a third agent, Charles, who believes that both sides of the box initially contained gas of the same temperature and pressure. Charles will take the removal of the partition to effect no change in macroscopically observable properties of the gas, and his credences may be represented by an equilibrium distribution, a probability measure that is invariant under dynamical evolution. If Alice and Bob are convinced that this distribution yields the same probabilities for results of macroscopic measurements as would their own credences, applied to the system, then they may use the equilibrium distribution - which will typically be much more tractable mathematically - as a surrogate for their own credences.

Taking statistical mechanical probabilities in this way removes some of the puzzles that have been associated with them. The equilibrium distribution distinguishes no direction in time. If we took it to represent Alice or Bob's credences about the state of the system at $t_{1}$, this would clash with their beliefs about the state of the system at $t_{0}$, as the equilibrium distribution at $t_{1}$ renders it overwhelmingly probable that the gas was spread evenly over the box at $t_{0}$. But this is not how it is being used; Alice and Bob are using it as a surrogate for the more complicated functions $\phi^{A}\left(t_{1}\right)$ and $\phi^{B}\left(t_{1}\right)$, and the justification for doing so is that the equilibrium distribution yields what are effectively the same probabilities for the results of measurements performed after $t_{1}$. There is no justification for applying this distribution to past events, and hence we do not encounter the disastrous retrodictions that prompt David Albert (2000) to introduce his Past Hypothesis.

\section{Chances, real or counterfeit?}

As mentioned, some philosophers might be willing to accept all the substantive claims made in this paper, yet resist the use of the word "chance" for the quantities we have discussed. Lewis himself might be among these; with reference to ideas advanced by Jeffrey $(1965, \S 12.7)$ and Skyrms $(1977,1980)$, Lewis speaks of a "kind of counterfeit chance" (Lewis, 1986, 120).

There is an argument, stemming from the Principal Principle, for the incompatibility of non-extremal chances with deterministic laws of nature (see Schaffer 2007, pp. 128-129). Recall that the PP says that a reasonable agent's credences should have it that, for any proposition $A$, any real number $\lambda$ in $[0,1]$, and any admissible information 
$E$,

$$
\operatorname{cr}(A \mid E \& \operatorname{ch}(A)=\lambda)=\lambda .
$$

Lewis does not offer a definition of admissibility, but he does declare that all propositions about past events and present states of affairs are admissible, "every detail—no matter how hard it might be to discover - of the structure of the coin, the tosser, other parts of the setup, and even anything nearby that might somehow intervene" (Lewis, 1980, p. 272). If the laws of nature are deterministic, then these laws, together with sufficient information about events to the past of $A$, entail either $A$ or its negation. Suppose that laws of nature are always admissible. This means that, for a suitable choice of admissible $E$, probabilistic coherence requires

$$
\operatorname{cr}(A \mid E)=0 \text { or } 1 .
$$

This in turn entails that our agent must assign zero credence to any proposition that asserts that the value of a chance lies in an interval not containing 0 or 1 . An agent whose credences satisfy Lewis' PP must be certain that, in a deterministic world, there are no non-extremal chances (that is, the agent must assign zero credence to the conjunction of some proposition that entails that the laws are deterministic and some proposition that entails that there are nonextremal chances). If we add the further condition, as Lewis (1980, p. 267) does, that the agent assign zero credence only to the empty proposition, true at no possible world, then the incompatibility of determinism and non-extremal chances follows. ${ }^{14}$

If it is part of our notion of chance that reasonable credences must satisfy Lewis' Principal Principle, with laws of nature and all propositions about the past of an event counted as admissible, then Lewis is right; determinism and chance are irreconcilable. This is a symptom of the fact that Lewis' notion of chance differs from the conception we are trying to capture in this paper. On Lewis' notion, chance requires chancy laws. The notion we are trying to capture stems from the idea that, in the face of unavoidable (or, perhaps, unavoidable for all practical purposes) limitations on accessible information about the world, there might be some credences that are optimal for an agent who makes maximal use of available information and dynamical features of the systems involved. There is nothing incoherent in Lewis' notion; indeed, as suggested by quantum mechanics, the fundamental laws of physics probably are chancy. We need not leave the notion of chance behind, however, when we emerge from the domain in which quantum chanciness predominates and enter into the realm of systems whose behaviour can be adequately modelled by classical mechanics. There is a useful conception of chance that is compatible with determinism.

A terminological distinction between the two notions is in order. Schaffer's term "epistemic chance" seems to be an apt one, as a term that combines epistemic and objective connotations. Lewisian metaphysicians may, if they choose, call such chances

\footnotetext{
${ }^{14}$ Note that, without this condition on credences, which Lewis calls regularity, nothing can follow about what chances actually are like from the PP, which is a condition on the credences of a reasonable agent, and hence can only tell us what a reasonable agent must believe.
} 
"counterfeit" chances, but we should not let this obscure the value that lies in the concept, nor should we let it dissuade us from accepting such chances as valid currency when appropriate. 


\section{References}

Albert, D. (2000). Time and Chance. Cambridge: Harvard University Press.

Cournot, A. A. (1843). Exposition de la Théorie des Chances et des Probabilités. Paris: Librairie de L. Hachette.

Daston, L. (1988). Classical Probability in the Enlightenment. Princeton: Princeton University Press.

de Finetti, B. (1937). La prevision: ses lois logiques, ses sources subjectives. Annales de l'Institute Henri Poincaré 7, 1-68. English translation in de Finetti (1980).

de Finetti, B. (1980). Foresight: its logical laws, its subjective sources. In H. E. Kyburg and H. E. Smokler (Eds.), Studies in Subjective Probability. Huntington, New York: Robert E. Krieger Publisher Company. Translation of de Finetti (1937).

Hacking, I. (1971). Equipossibility theories of probability. British Journal for the Philosophy of Science 22, 339-355.

Hacking, I. (1975). The Emergence of Probability. Cambridge: Cambridge University Press.

Howie, D. (2002). Interpreting Probability: Controversies and Developments in the Early Twentieth Century. Cambridge: Cambridge University Press.

Jeffrey, R. C. (1965). The Logic of Decision. New York: McGraw-Hill.

Laplace, P.-S. (1814). Essai Philosophique sur les Probabilités. Paris: Courcier. English translation in Laplace (1951).

Laplace, P.-S. ([1902] 1951). A Philosophical Essay on Probabilities. New York: Dover Publications. Translation of Laplace (1814).

Lewis, D. (1980). A subjectivist's guide to objective chance. In R. C. Jeffrey (Ed.), Studies in Inductive Logic and Probability, Volume II, pp. 263-93. University of Caifornia Press.

Lewis, D. (1986). Postscripts to "A subjectivist's guide to objective chance". In Philosophical Papers, Volume II. Oxford: Oxford University Press.

Machina, M. J. (2004). Almost-objective uncertainty. Economic Theory 24, 1-54.

Poincaré, H. ([1905] 1952). Science and Hypothesis. Dover Publications.

Poincaré, H. (1912). Calcul des probabilités. Paris: Gauthier-Villars. 
Poisson, S.-D. (1837). Recherches sur la Probabilité des Jugements en Matière Criminelle et en Matière Civile, précédées des règles générales du calcul des probabilités. Paris: Bachelier, Imprimeur-Libraire.

Popper, K. R. (1957). The propensity interpretation of the calculus of probability, and the quantum theory. In S. Körner (Ed.), Observation and Interpretation: a symposium of philosophers and physicists, pp. 65-70. London: Butterworths.

Popper, K. R. (1959). The propensity interpretation of probability. The British Journal for the Philosophy of Science 37, 25-42.

Savage, L. J. ([1954] 1972). The Foundations of Statistics. New York: Dover Publications, Inc.

Savage, L. J. (1973). Probability in science: A personalistic account. In P. Suppes (Ed.), Logic Methodology, and Philosophy of Science IV, pp. 417-428. Amsterdam: North-Holland.

Schaffer, J. (2007). Deterministic chance? The British Journal for the Philosophy of Science 58, 113-140.

Shimony, A. (1971). Scientific inference. In R. Colodny (Ed.), The Nature and Function of Scientific Theories, pp. 79-172. Pittsburgh: Pittsburgh University Press. Reprinted in Shimony (1993).

Shimony, A. (1993). Seach for a Naturalistic World View, Volume I: Scientific Method and Epistemology. Cambridge University Press.

Skyrms, B. (1977). Resiliency, propensities, and causal necessity. Journal of Philosophy 74, 704-713.

Skyrms, B. (1980). Causal Necessity. New Haven, CT.

Strevens, M. (1999). Objective probability as a guide to the world. Philosophical Studies 95, 243-275.

von Plato, J. (1983). The method of arbitrary functions. The British Journal for the Philosophy of Science 34, 37-47. 